\title{
Raman spectroscopy of epitaxial InGaN/Si in the central composition range
}

Mani Azadmand ${ }^{1 *}$, Emiliano Bonera ${ }^{1}$, Daniel Chrastina ${ }^{2}$, Sergio Bietti ${ }^{1}$, Shiro Tsukamoto ${ }^{1}$, Richard Nötzel $^{3}$, and Stefano Sanguinetti ${ }^{1}$

${ }^{1}$ L-NESS and Dipartimento di Scienza dei Materiali, Università di Milano - Bicocca, Milano (Italy)

${ }^{2}$ L-NESS and Dipartimento di Fisica, Politecnico di Milano, Como (Italy)

${ }^{3}$ Academy of Advanced Optoelectronics, South China Normal University, Guangzhou (China)

*Email: m.azadmand@campus.unimib.it

\begin{abstract}
InGaN alloys are of raising interest for many applications. The possibility of tuning their functional properties with the composition sets the importance of finding methods to characterize these materials in a fast and non-destructive way. Raman spectroscopy is one of these techniques, being able to yield information about the composition, strain, and other relevant parameters. However, the method of measuring the composition with a calibration of the maximum $A_{1}(L O)$ band is today limited to regions which are either rich in In or in Ga. The middle composition range still needs a calibration. By measuring the Raman spectra of different $\mathrm{In}_{\mathrm{x}} \mathrm{Ga}_{1-\mathrm{x}} \mathrm{N}$ alloys grown epitaxially on $\mathrm{Si}$ and by comparing them with the results from $x$-ray diffraction, we investigated this missing region of compositions. Within the range of $30 \%$ to $65 \%$, we have found that the position of the maximum of $A_{1}(\mathrm{LO})$ scales with the In fraction $x$ as $\omega_{\mathrm{x}}=736-135 x-24 x^{2} \mathrm{~cm}^{-1}$. With this calibration, it is possible to determine, by Raman spectroscopy, the composition of an unknown alloy with an uncertainty of $5 \%$.
\end{abstract}

\section{Introduction}

InGaN has drawn much attention in applications like optoelectronics and hydrogen generation by water splitting ${ }^{1-4}$. The substitution of In and Ga atoms in InGaN alloys has a considerable influence on optical and electronic properties of the material since there is a large difference between the bandgap of $\mathrm{InN}(0.7 \mathrm{eV})$ and $\mathrm{GaN}(3.42 \mathrm{eV}) .{ }^{5}$ For this reason, 
the compositional control of InGaN is of great importance. X-ray diffraction (XRD) and photoluminescence (PL) have been used as the main characterization methods to define the composition of InGaN alloys. ${ }^{6-8}$ However, there are some limitations, especially in case of PL which is only useable for very high quality $\mathrm{InGaN}$ (grown at elevated temperatures above $\left.650{ }^{\circ} \mathrm{C}\right)^{9}$. XRD is very robust, but it is unable to probe locally with a resolution better than one micrometer unless using a synchrotron source.

These limitations can be overcome by using Raman spectroscopy, which is a non-destructive method that gives the possibility to probe the sample locally with a lab-scale compatible equipment. Moreover, this method is very fast and simpler to interpret than PL and XRD to determine the In amount in the InGaN alloys. For example the interpretation of PL can be complicated by the diffusion of the photogenerated carriers.

In the literature there are already studies on the determination of composition of InGaN by Raman spectroscopy, and several authors ${ }^{10-15}$ addressed the interpretation of the Raman spectra, for example in terms of polarization response and resonance effects. However, these works lack of data in the middle composition range (between $30 \%$ and $65 \%$ In).

Obtaining $\operatorname{In}_{x} \mathrm{Ga}_{1-\mathrm{x}} \mathrm{N}$ crystals with high In amount is difficult, as a result of low dissociation temperature of $\mathrm{InN}$ and high equilibrium vapor pressure of nitrogen. The different binding energies of In-N and Ga-N are reflected in the different decomposition temperatures ${ }^{16}$. Lowtemperature growth has been used to avoid it. ${ }^{17-20}$. However, in low-temperature growth the formation of metal droplets on the surface drastically reduces the Indium incorporation in the crystal ${ }^{21,22}$ and causes a vertical compositional gradient. ${ }^{22}$ We have previously shown ${ }^{23}$ the effect of $(\mathrm{In}+\mathrm{Ga}) / \mathrm{N}$ ratio on the formation of metal droplets and consequently depleting metal adatoms on the surface.

By growing $\mathrm{InGaN}$ in the low-temperature regime and by a careful control of the growth parameters to avoid formation of metal droplets on the surface, a good composition control is achievable. We used such high quality $\mathrm{InGaN}$ layers to fill the gap in Raman spectroscopy data in the middle composition range, thus providing a useful reference for the determination of the composition of this alloy by Raman spectroscopy.

\section{Methods}

The $\operatorname{In}_{x} \mathrm{Ga}_{1-\mathrm{x}} \mathrm{N}$ (x varying between 0-0.65) samples were grown on 2-inch $\mathrm{Si}(111)$ wafers, using plasma-assisted molecular beam epitaxy (PAMBE). The native oxide layers were removed from the Si surface by a thermal annealing at $900{ }^{\circ} \mathrm{C}$ for 20 mins in the growth 
chamber. The clean silicon $7 \times 7$ surface reconstruction was observed using in-situ reflection high-energy electron diffraction (RHEED). This was used also for the calibration of substrate temperature $\left(860{ }^{\circ} \mathrm{C}\right)^{24}$. Prior to $\mathrm{InGaN}$ growth, the substrates were exposed to a nitrogen plasma with flux of 0.9 standard cubic centimeter per minute $(\mathrm{sccm})$ and RF power of 250 $\mathrm{W}$ at $850{ }^{\circ} \mathrm{C}$ for 5 minutes. This intentional nitridation process is known to improve the epitaxial relation of (In)GaN epilayers with the Si substrate ${ }^{3,25}$. The same plasma parameters also are used to grow the InGaN thin-films. A total thickness of about $100 \mathrm{~nm}$ was deposited. To avoid metal droplets formation on the surface which causes compositional fluctuations during the growth, the growth was performed in the N-rich $((\mathrm{In}+\mathrm{Ga}) / \mathrm{N}<1)$ regime. In order to increase the Indium incorporation in the crystal and better control the composition tunability, all InGaN samples were grown at low growth temperature $\left(450{ }^{\circ} \mathrm{C}\right)$, below the $\mathrm{InN}$ dissociation temperature ${ }^{26}$. For each sample, the $\mathrm{In} / \mathrm{Ga}$ ratio was carefully changed to obtain $\operatorname{In}_{\mathrm{x}} \mathrm{Ga}_{1-\mathrm{x}} \mathrm{N}$ with different amount of In.

After the growth, the center of the samples was used for characterization. A PANalytical X'Pert PRO high-resolution diffractometer was used to perform X-ray diffraction (XRD) measurements. A hybrid mirror and 2-bounce Ge monochromator selected the Kal line, $\lambda=0.15406 \mathrm{~nm}$, of the $\mathrm{Cu}$ anode. A high-precision goniometer allowed both translational $(x, y$ and $z$ ) and rotational (incidence angle $\omega$, diffraction angle $2 \theta$, sample rotation $\varphi$ and sample tilt $\chi$ ) degrees of freedom. A three-bounce Ge monochromator placed in front of the detector was used as an analyser crystal, in order to obtain high precision in $2 \theta$ and to reject fluorescence from the sample. To assess the composition, a $\omega-2 \theta$ scan through the InGaN(0002) Bragg peak was carried out, using the $\mathrm{Si}(111)$ peak from the substrate as a reference. The results can be found in Fig. 1 where the InGaN compositional dependence shift is shown. Asymmetric reciprocal space maps demonstrated that the material was fully relaxed and maintained an epitaxial relationship with the substrate.

Raman spectra were collected at room temperature by a T64000 Jobin Yvon spectrometer in a single spectrometer configuration. The excitation source was a $532 \mathrm{~nm}$ laser focused by a 100x 0.90 NA objective with about $1 \mathrm{~mW}$ excitation power and $700 \mathrm{~nm}$ spot size on the sample. The power density was checked not to introduce changes in the spectrum due to lowering of the bands due to laser heating. The configuration is in backscattering and the collection is kept without polarizers. Typical Raman spectra can be found in Fig. 1 where it is possible to observe the presence of the contribution of the epilayer together with the signal from the Si substrate. 


\section{Results and discussion}

The XRD measurements shown in Fig. 1 demonstrate a progression to lower $\theta$ values as the In content increases, corresponding to an increase in the $c$ parameter of the lattice. The mismatch between the in-plane InGaN lattice parameter $a$ and the relevant in-plane spacing of the $\mathrm{Si}(111)$ surface is very large at about $-13 \%$ to $-17 \%$ that no residual strain is expected, and indeed a reciprocal space map around $\operatorname{Si}(224)$ and $\operatorname{InGaN}(1015)$ for a sample with $x=$ $39 \%$ showed full relaxation. This means that the $c$ parameter alone can be used in order to ascertain the In content. While XRD itself is a high-precision technique (especially if the substrate can be used as a reference) the accuracy here is limited by the use of a linear interpolation between the lattice parameters of $\mathrm{InN}$ and $\mathrm{GaN}$, and by a possible nonuniformity in the deposited material.

Care must be taken therefore to ensure that the micro-Raman laser spot illuminates a representative region of the material. For this reason the same piece of samples which were characterized by XRD, are also used for Raman spectroscopy. Moreover, different spots on these pieces of samples are examined by micro-Raman to confirm the independency of the results with respect to the position of the laser spot on the samples. Figure 1 shows a set of bands in the longitudinal optical region for concentrations ranging from $31 \%$ to $65 \%$. The spectra are normalized to maximum amplitude in order to highlight the shape and position of the spectra. These spectra are in the region of the $A_{1}(L O)$ band of $I n N$ and GaN. The $E_{2}$ bands, expected around 500 and $550 \mathrm{~cm}^{-1}$, could not be unambiguously detected because of the strong scattering of the silicon background. The main features of the Raman spectra of the InGaN alloys shown in Fig. 1 could also be affected by the appearance at frequencies lower than $A_{1}$ of a shoulder which has been tentatively attributed to a disordered activated $\mathrm{B}_{1}$-like mode ${ }^{15,27}$, indicated also as the $\mathrm{S}$-band. For most of the samples the $\mathrm{A}_{1}$ and $\mathrm{B}_{1}$ peaks are clearly distinguishable due to the higher intensity of $A_{1}$ mode. Hence, no considerable influence (considering the error bar) on determination of the $A_{1}$ peak position is expected. 


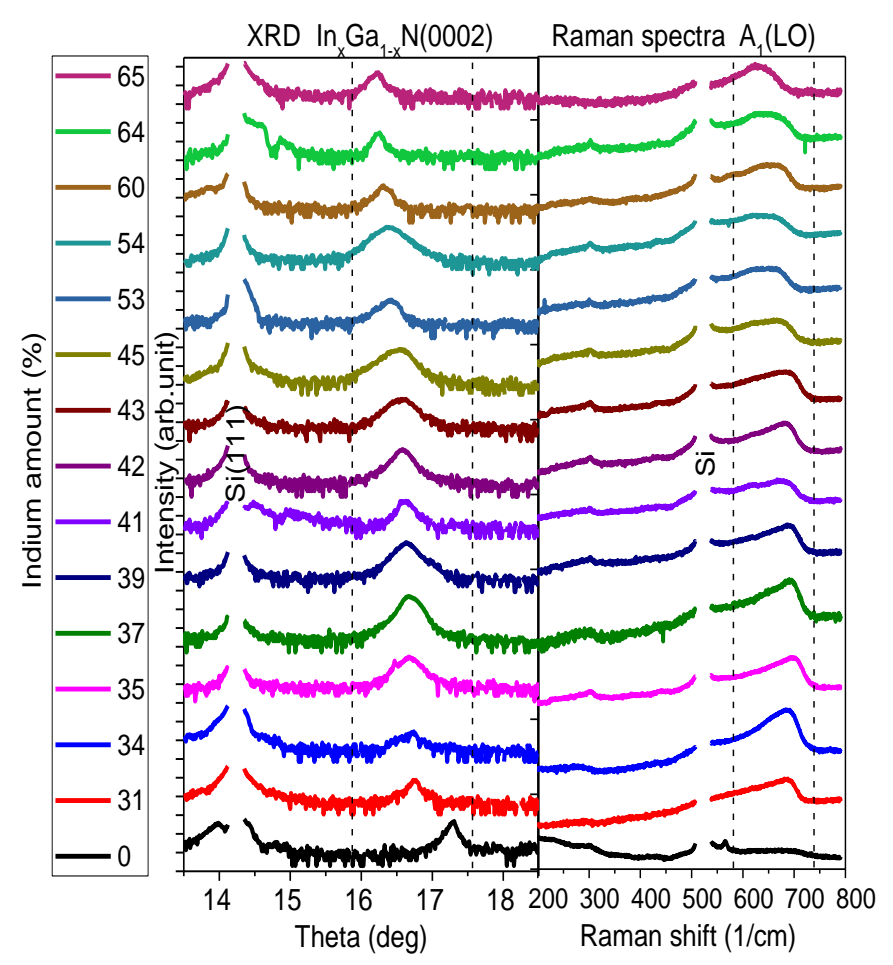

Fig. 1: $\omega-2 \theta$ scan XRD (left) and room-temperature Raman spectra (right), of a set of wurtzite $\mathrm{In}_{\mathrm{x}} \mathrm{Ga}_{1-\mathrm{x}} \mathrm{N}$ epilayers (grown on $\mathrm{Si}$ ) with Indium fractions ranging from $\mathrm{x}=0$ up to 0.65 .

The bands are rather broad, but this is a typical feature of InGaN alloys. The large width of the bands is consistent with the previous results on these alloys in the literature (see for example ref. [Davydov et al.] ${ }^{10}$ ) and it is a result of the fact that the material is a ternary alloy, and therefore due to alloy disorder the selection rules for $\mathrm{InN}$ or $\mathrm{GaN}$, requiring a scattering only for the center-zone phonon, are relaxed. This effect is quite evident considering also the asymmetry of the broadening towards low energies. Figure 2 shows the full-width at half-maximum (FWHM) of the InGaN LO bands and (0002) Bragg peaks as a function of the In concentration. In case of InGaN (0002) Bragg peaks, although the use of the analyzer crystal removes the broadening of the InGaN peak which arises from misorientation of the (0002) planes with respect to the sample surface (or from the limited lateral crystalline domain size), both vertical and lateral inhomogeneity lead to a broadening of the average diffraction peaks from the material probed by the x-ray beam. The FWHM of both XRD and Raman peaks reach to its maximum where the In concentration is close to $50 \%$ (maximum immiscibility of $\mathrm{InN}$ and $\mathrm{GaN}$ ) and reduces by going to lower or higher concentrations. It worth to mention that the FWHM of Raman spectra can be notably affected by the observed $\mathrm{B}_{1}$ bands at the shoulder of $\mathrm{A}_{1}$ bands. 


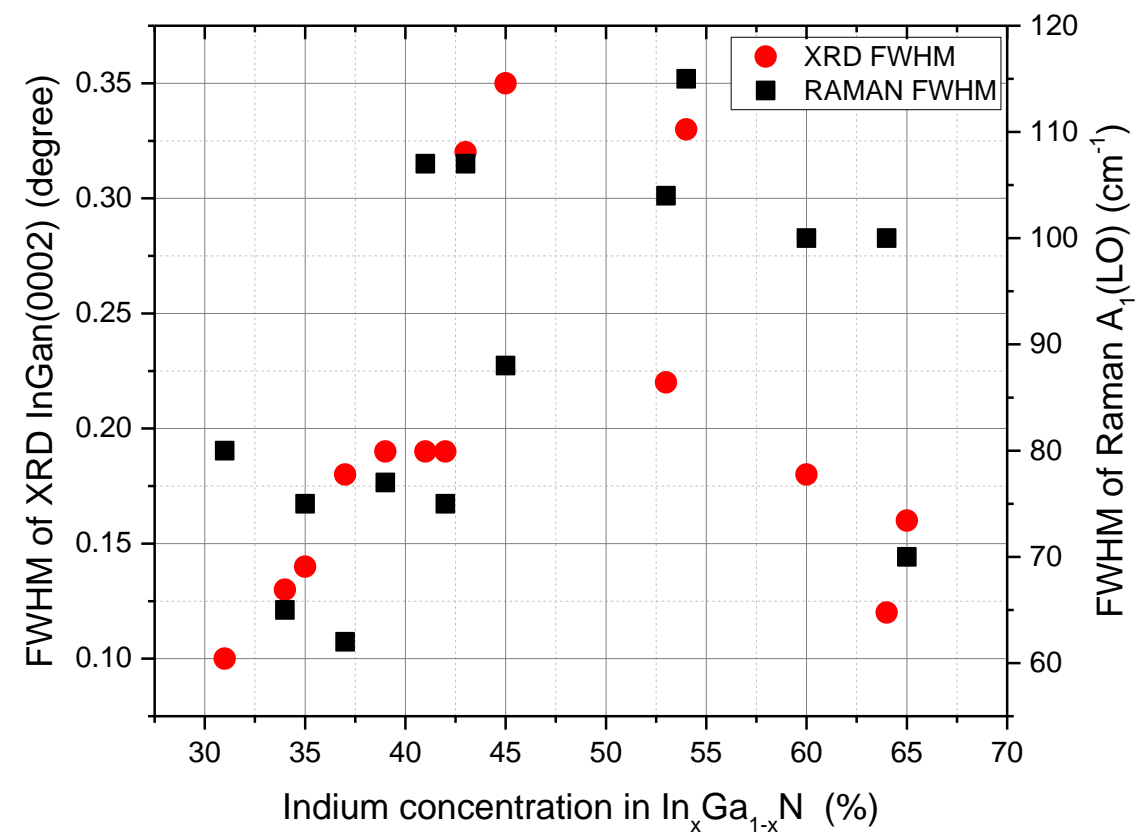

Fig. 2: FWHM of the LO bands as a function of the In concentration

Although the bands are relatively broad, the composition can be neatly followed by a Polynomial dependence of the maxima with respect to the concentration. Figure 3 shows the shift of the $A_{1}$ band as a function of the Indium concentration $\mathrm{x}$. In this method we used the centroid (the maximum) of the bands. Notice the uncertainties reported in this plot for the Raman shift do not indicate the FWHM of the bands ${ }^{12}$, but rather to the precision in the determination of the maxima. The uncertainty is represented in Fig. 3 by the finite size of the dots, and it has been determined by repeating the measurements several times on several positions on the samples.

The polynomial fit of the dependence of the Raman $A_{1}(L O)$ frequency on the In composition, based on the results of this study (central composition range) including the known values of the $\mathrm{InN}$ and $\mathrm{GaN}$ ( $\mathrm{GaN}$ is used as a fix point) binary compounds is (see Fig. 3):

$$
\omega_{\mathrm{x}}=\left(736-135 x-24 x^{2}\right) \mathrm{cm}^{-1}
$$

Not all the points intersect the fitted line, but they do by rigidly shifting the curve in the horizontal axis by about $\pm 5 \%$. We called this value the uncertainty in the determination of the relative concentration of In which is related to the broadness of the bands. Another reason for the observed scattering could be the presence of the $\mathrm{B}_{1}$ band. In some cases, when the 
effect of the $\mathrm{B}_{1}$ band is important, the overall effect is to shift the maximum of the broad $\mathrm{A}_{1}$ band towards lower wavenumbers.

In addition to the linear dependence of $\mathrm{A}_{1}(\mathrm{LO})$ with In fraction, observed experimentally in literature in the case of Ga-rich and In-rich InGaN $^{11-14}$ as well as theoretically predicted by the modified random-element isodisplacement (MREI) model ${ }^{27}$, a bowing toward higher frequencies in the middle region is observed in this study. Likewise, Kim et al. ${ }^{15}$ observed a large bowing respect to theoretical predictions, in the case of $\mathrm{E}_{2}$ (high) mode of $\operatorname{In}_{\mathrm{x}} \mathrm{Ga}_{1-\mathrm{x}} \mathrm{N}$ alloys in the middle composition range. In the case of $\mathrm{A}_{1}(\mathrm{LO})$ mode, although an upward shift with respect to predicted values (linear fit) was observed in the litratures ${ }^{11,12,28}$, it was attributed to residual compressive strain of the epilayers. However, as it was mentioned earlier, asymmetric reflections in reciprocal space map XRD confirm full relaxation of the epilayers grown in the current study which suggests the previous assumption of linear dependency of $\mathrm{A}_{1}(\mathrm{LO})$ with $\mathrm{In}$ fraction in the entire InGaN compositional range must be reconsidered.

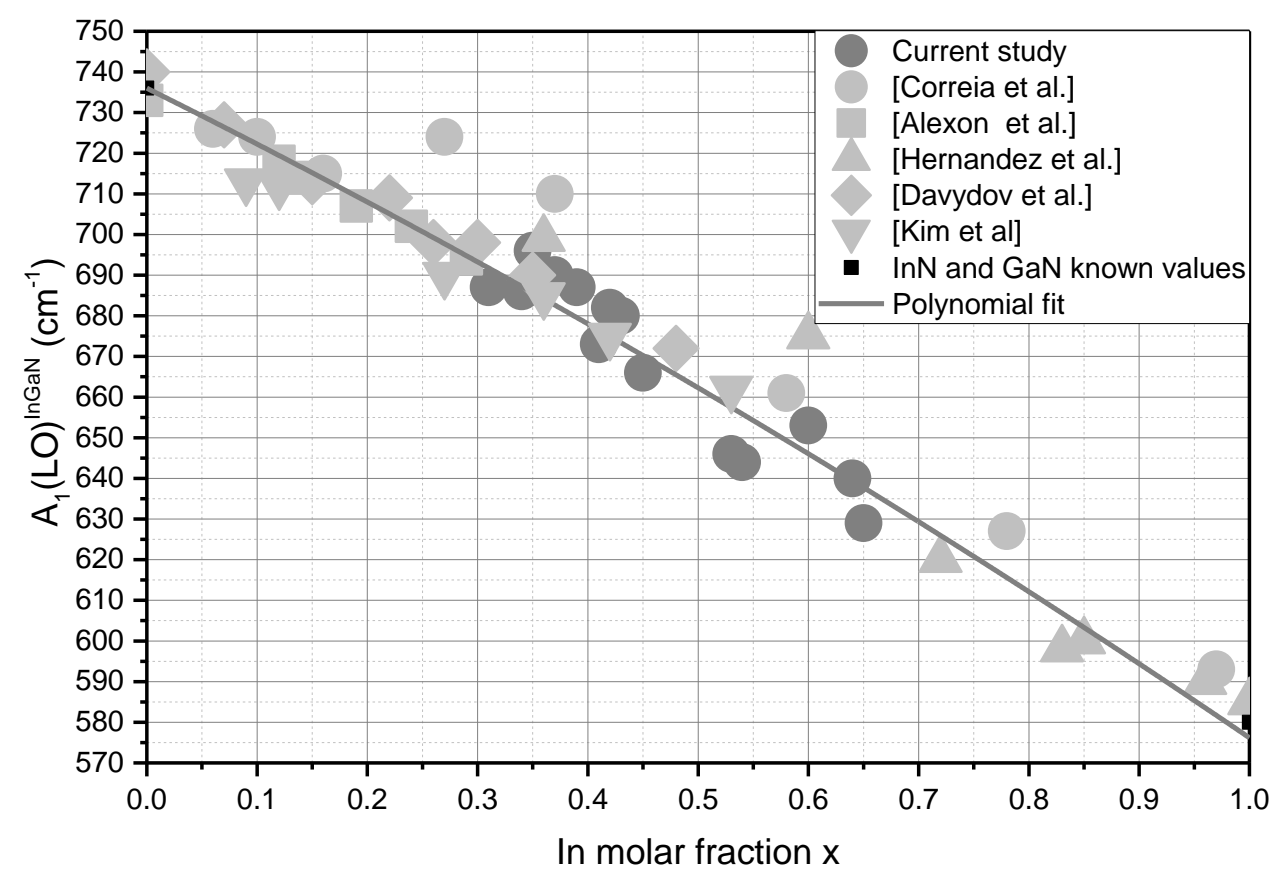

Figure 3: Raman shift as a function of the In concentration, for the entire InGaN composition range based on the data measured in the middle composition range (current study), Correia et al. ${ }^{11}$, Alexson et al. ${ }^{13}$, Hernandez et al. ${ }^{12}$, Davydov et al. ${ }^{10}$, Kim et al. ${ }^{15}$, and known values for $\mathrm{GaN}\left(736 \mathrm{~cm}^{-1}\right)^{29}$ and $\mathrm{InN}\left(580 \mathrm{~cm}^{-1}\right)^{30}$. The Polynomial fit based on Eq. (1) (the results of this study and known values for $\mathrm{GaN}$ and $\mathrm{InN}$ ) is plotted as a solid line. 


\section{Conclusions}

We have found that in contrary to the linear dependence of $A_{1}(\mathrm{LO})$ with In fraction reported in the literature, in the central zone of $\mathrm{InGaN}$ it follows a polynomial relationship with a bowing toward higher frequencies in the middle region. These results complete the available literature and add a convenient method for the determination of local properties of InGaN films and nanostructures within this region of composition.

\section{References}

${ }^{1}$ T. Wang, J. Bai, S. Sakai, and J.K. Ho, Appl. Phys. Lett. 78, 2617 (2001).

${ }^{2}$ N. ul H. Alvi, P.E.D. Soto Rodriguez, P. Aseev, V.J. Gómez, A. ul H. Alvi, W. ul Hassan, M. Willander, and R. Nötzel, Nano Energy 13, 291 (2015).

${ }^{3}$ P. Aseev, P.E.D.S. Rodriguez, V.J. Gómez, N.U.H. Alvi, J.M. Mánuel, F.M. Morales, J.J. Jiménez, R. García, A. Senichev, C. Lienau, E. Calleja, and R. Nötzel, Appl. Phys. Lett. 106, 072102 (2015).

${ }^{4}$ N.H. Alvi, P.E.D. Soto Rodriguez, P. Kumar, V.J. Gómez, P. Aseev, A.H. Alvi, M.A. Alvi, M. Willander, and R. Nötzel, Appl. Phys. Lett. 104, (2014).

${ }^{5}$ F.K. Yam and Z. Hassan, Superlattices Microstruct. 43, 1 (2008).

${ }^{6}$ T. Kuykendall, P. Ulrich, S. Aloni, and P. Yang, Nat. Mater. 6, 951 (2007).

7 Ž. Gačević, V.J. Gómez, N. García Lepetit, P.E.D. Soto Rodríguez, A. Bengoechea, S. Fernández-Garrido, R. Nötzel, and E. Calleja, J. Cryst. Growth 364, 123 (2013).

${ }^{8}$ M. Schuster, P.O. Gervais, B. Jobst, W. Hösler, R. Averbeck, H. Riechert, A. Iberl, R. Stömmer, O. Ambacher, and J. Majewski, J. Phys. D. Appl. Phys. 32, A56 (1999).

${ }^{9}$ N. Yoshimoto, T. Matsuoka, T. Sasaki, and A. Katsui, Appl. Phys. Lett. 59, 2251 (1991).

${ }^{10}$ V.Y. Davydov, A.A. Klochikhin, V. V. Emtsev, A.N. Smirnov, I.N. Goncharuk, A. V. Sakharov, D.A. Kurdyukov, M. V. Baidakova, V.A. Vekshin, S. V. Ivanov, J. Aderbold, J. Graul, A. Hashimoto, and A. Yamamoto, Phys. Status Solidi Basic Res. 240, 425 (2003).

${ }^{11}$ M.R. Correia, S. Pereira, E. Pereira, J. Frandon, E. Alves, M.R. Correia, S. Pereira, and E. Pereira, Appl. Phys. Lett. 83, 4761 (2003).

${ }^{12}$ S. Hernandez, R. Cusco, D. Pastor, L. Artus, K.P. O’Donnell, R.W. Martin, I.M. Watson, Y. Nanishi, J. Appl. Phys. 98, 0 (2005).

${ }^{13}$ D. Alexson, L. Bergman, R.J. Nemanich, M. Dutta, M.A. Stroscio, C.A. Parker, F. Adar, J. Appl. Phys. 241, (2012).

${ }^{14}$ R. Oliva, J. Ibanez, R. Cusco, R. Kudrawiec, J. Serafinczuk, O. Martinez, J. Jimenez, M. Henini, C. Boney, A. Bensaoula, and L. Artus, J. Appl. Phys. 1, 0 (2012).

15 J.G. Kim, Y. Kamei, A. Kimura, N. Hasuike, H. Harima, K. Kisoda, T. Hotta, K. Sasamoto, and A. Yamamoto, Phys. Status Solidi Curr. Top. Solid State Phys. 9, 730 (2012).

${ }^{16}$ O. Ambacher, J. Vac. Sci. Technol. B Microelectron. Nanom. Struct. 14, 3532 (1996).

${ }^{17}$ P. Aseev, P.E.D.S. Rodriguez, P. Kumar, V.J. Gómez, N.U.H. Alvi, J.M. Mánuel, F.M. Morales, J.J. Jiménez, R. García, E. Calleja, and R. Nötzel, Appl. Phys. Express 6, 115503 (2013). 
${ }^{18}$ P. Kumar, P.E.D.S. Rodriguez, V.J. Gómez, N.H. Alvi, E. Calleja, and R. Nötzel, Appl. Phys. Express 6, 035501 (2013).

${ }^{19}$ C.A.M. Fabien, B.P. Gunning, W. Alan Doolittle, A.M. Fischer, Y.O. Wei, H. Xie, and F.A. Ponce, J. Cryst. Growth 425, 115 (2015).

${ }^{20}$ T. Yamaguchi, N. Uematsu, T. Araki, T. Honda, E. Yoon, and Y. Nanishi, J. Cryst. Growth 377, 123 (2013).

21 M.A.L. Johnsona, W.C. Hughes, W.H. Rowland, J.W. Cook, J.F. Schetzinaa, M. Leonardd, H.S. Kong ", J.A. Edmond ', and J. Zavada, J. Cryst. Growth 175, 76 (1997).

${ }^{22}$ T. Böttcher, S. Einfeldt, V. Kirchner, S. Figge, H. Heinke, D.H. Selke, P.L. Ryder, S. Einfeldt, V. Kirchner, S. Figge, H. Heinke, D. Hommel, T. Bo, H. Selke, and P.L. Ryder, Appl. Phys. Lett. 3232, 3232 (1998).

${ }^{23}$ M. Azadmand, L. Barabani, S. Bietti, D. Chrastina, E. Bonera, M. Acciarri, A. Fedorov, S. Tsukamoto, R. Nötzel, and S. Sanguinetti, Sci. Rep. 8, 11278 (2018).

${ }^{24}$ K. Yokota, S. Tamura, D. Huang, H. Uchida, M. Aono, M. Sakai, and M. Suemitsu, Jpn. J. Appl. Phys. 32, L610 (1993).

${ }^{25}$ Y. Nakada, I. Aksenov, and H. Okumura, Appl. Phys. Lett. 73, 827 (1998).

${ }^{26}$ R. Averbeck and H. Riechert, Phys. Status Solidi Appl. Res. 176, 301 (1999).

${ }^{27}$ H. Grille and F. Bechstedt, Phys. Rev. B 61, 20 (2000).

${ }^{28}$ M.R. Correia, S. Pereira, E. Pereira, J. Frandon, I.M. Watson, C. Liu, E. Alves, A.D. Sequeira, and N. Franco, Appl. Phys. Lett. 85, 2235 (2004).

${ }^{29}$ T. Kozawa, T. Kachi, H. Kano, Y. Taga, and M. Hashimoto, J. Appl. Phys. 1098, (2012).

${ }^{30}$ T. Inushima, T. Shiraishi, and V.Y. Davydov, Solid State Commun. 110, 491 (1999). 